\title{
Choice by lexicographic semiorders
}

\author{
Paola Manzini \\ School of Economics and Finance, University of St. Andrews and IZA \\ Marco Mariotti \\ School of Economics and Finance, University of St. Andrews
}

\begin{abstract}
In Tversky's (1969) model of a lexicographic semiorder, a preference is generated via the sequential application of numerical criteria by declaring an alternative $x$ better than an alternative $y$ if the first criterion that distinguishes between $x$ and $y$ ranks $x$ higher than $y$ by an amount exceeding a fixed threshold. We generalize this idea to a fully fledged model of boundedly rational choice. We explore the connection with sequential rationalizability of choice (Apesteguia and Ballester 2010, Manzini and Mariotti 2007) and we provide axiomatic characterizations of both models in terms of observable choice data.

KeYwords. Lexicographic semiorders, bounded rationality, revealed preference, choice.
\end{abstract}

JEL CLASSIFICATION. D0.

\section{INTRODUCTION}

Lexicographic heuristics have gained much attention in the study of decision making in several fields: in psychology (e.g., Tversky 1969, 1972, Gigerenzer and Todd 1999); in positive economics (e.g., Rubinstein 1988, Leland 1994, Manzini and Mariotti 2007, Apesteguia and Ballester 2010); in normative economics (e.g., Tadenuma 2002, 2005; Houy and Tadenuma 2009); in marketing science (e.g., Kohli and Jedidi 2007, Yee et al. 2007). Medina et al. (2011) note that the Talmud contains arguments in favor of a lexicographic ranking of the rationales used to adjudicate between pairs of alternatives.

The explanation for this success is obvious: lexicographic procedures look appealingly simple and realistic since they eschew the complex trade-offs between several criteria of classical decision makers. Alternatively, the lack of trade-offs may also seem to

Paola Manzini: paola.manzini@st-andrews.ac.uk

Marco Mariotti: marco.mariotti@st-andrews.ac.uk

Countless discussions on related matters with Michael Mandler were crucially helpful in shaping our ideas for this paper. We were also influenced by a previous version of Jose Apesteguia and Miguel Angel Ballester's (2010) paper and by useful exchanges with them, for which we are grateful. We also thank the coeditor, Bart Lipman, two anonymous referees, and Sean Horan for many insightful comments. Finally, we are grateful to Gil Riella for spotting a mistake in a previous version of this paper. Part of the work on this paper was carried out while visiting the Economics Department "Marco Fanno" at the University of Padova; their generous hospitality is gratefully acknowledged.

Copyright $\odot 2012$ Paola Manzini and Marco Mariotti. Licensed under the Creative Commons AttributionNonCommercial License 3.0. Available at http: //econtheory . org.

DOI: 10.3982/TE679 
constitute a disadvantage (especially among economists). Price may be the most important criterion in the purchase of a house from a set of suitable houses. Yet who would be prevented by a difference of a few bucks from selecting a house in a much more desirable neighborhood? Arguably, very few people would be so uncompromising as to ignore any significant improvement in one dimension because of an arbitrarily small loss in the most important dimension. When modelling boundedly rational behavior, the rigid application of simple "rules of thumb" (such as buy the cheapest house among the acceptable ones) may look even less realistic than the trade-offs of textbook utility maximization.

In other words, it is reasonable that, even in a boundedly rational heuristic, criteria that detect significant differences between the alternatives under consideration should override criteria that do not. In this paper we study a model of choice that formalizes this intuition. Note that a number of "basic criteria" could be aggregated into a single, more complex criterion to which our observations on the house buyer above would nevertheless still apply: if the agent constructs an index which trades off price and location, that index constitutes a new criterion, for which it may be unwise not to ignore small differences in favor, say, of house size and so on. ${ }^{1}$ Only a fully rational decision maker would be able to pack together all possible trade-offs in a single criterion. However, in a more realistic model of decision making, there is a limit to the number of simultaneous trade-offs the decision maker is able to carry out. Thus, it seems plausible to expect the decision maker to rely on a lexicographic list of "slack" criteria. The choice procedure we propose can explain observed "anomalies," while at the same time preserving a convincing flexibility.

Considerations of this kind have already led some of the researchers mentioned above $^{2}$ to build models of preference or binary choice based on the application of numerical criteria where small differences in the values of criteria are ignored. ${ }^{3}$ However, such models leave unanswered the issue of choice from more complex sets (e.g., budget sets). They do not study choice functions. If binary preferences are derived from a boundedly rational procedure, the issue of associating such preferences with higher order choices is far from trivial: on the one hand, it may be impossible to maximize the preference (when it is cyclical); on the other hand, it may be inappropriate to even consider maximization when the issue is one of bounded rationality.

We focus on Tversky's (1969) fruitful notion of lexicographic semiorder, in which a preference is generated by the sequential application of numerical criteria by declaring an alternative $x$ better than an alternative $y$ if the first criterion that distinguishes between $x$ and $y$ ranks $x$ higher than $y$ by an amount exceeding a fixed threshold. Our first contribution is to define a choice procedure (choice by lexicographic semiorder) based on Tversky's idea.

\footnotetext{
${ }^{1}$ As another example, in Manzini and Mariotti (2006) we propose a multicriterion model of choice over time in which the first criterion is the exponentially discounted value, which trades off the time and size of a monetary reward.

${ }^{2}$ Tversky (1969), Rubinstein (1988), and Leland (1994).

${ }^{3} \mathrm{~A}$ difference being small is often interpreted as "similarity."
} 
Tversky himself considered lexicographic semiorders appealing but restrictive as a model of preference. ${ }^{4}$ In fact, this judgement is shown to be somewhat pessimistic. Even when the agent is endowed with very rudimental discriminatory abilities (being only able to classify criteria values in "good," "neutral," and "bad," where just good and bad are rankable), the model can account for a very rich variety of behaviors. In particular, when only binary choices are involved (as, for example, in several voting models ${ }^{5}$ ), the model is shown to be completely unrestrictive, provided that the set of alternatives is not too large (Proposition 1). More generally, the model can explain any set of choice data satisfying the weak axiom of revealed preferences (WARP) (Proposition 2): since such choices may not satisfy the strong axiom of revealed preferences (SARP), they may be highly "irrational" in that they exhibit strict revealed preference cycles.

The model turns out to be connected with another, much more general looking, notion of boundedly rational choice, namely sequentially rationalizable choice (Manzini and Mariotti 2007): an arbitrary number of arbitrary asymmetric binary relations (rationales) is applied sequentially to single out an alternative. On any finite domain, ${ }^{6}$ the lexicographic semiorder model restricts choice data the same way as the sequential rationalizability model under the additional assumption that the rationales used in the latter are acyclic (Proposition 3).

We note, however, that the clause "on any finite domain" is key. When this clause is relaxed, even marginally, by allowing a countably infinite number of finite choice sets, the equivalence breaks down in a major way: even the use of only two rationales may produce behaviors that cannot be generated by any number of semiorders and any number of discriminations (Proposition 4). So, the two models are, in general, clearly distinct.

Next, we characterize choice by lexicographic semiorders in terms of a new contraction consistency condition (Reducibility), at the same time providing an algorithm to construct the semiorders (Theorem 1).

Our technique leads straightforwardly to a relaxation of Reducibility which characterizes sequential rationalizability (Theorem 2 ). This result, while quite tangential to the main line of enquiry of this paper, is of independent interest, since the characterization of sequential rationalizability proves to be a hard problem, which we left open in Manzini and Mariotti (2007). Our results in this respect build on and complement those by Apesteguia and Ballester (2010), who are the first to draw attention to the restriction of sequential rationalizability to acyclic rationales and to provide a characterization for it on finite domains. In the Appendix, we work out one of their examples of sequentially rationalizable choices to construct the rationales with our algorithm. Our work can also be fruitfully seen as an extension of the approach in Mandler et al. (2012): we discuss this relation in the concluding section.

\footnotetext{
${ }^{4}$ See Section 3.

${ }^{5}$ See, e.g., Kalandrakis (2010) and the references therein for a recent example. We discuss Kalandrakis' work more fully in Section 3.1.

${ }^{6}$ That is, a domain including a finite number of finite sets.
} 


\section{Lexicographic semiorders: Preferences ANd CHOICE}

Fix a nonempty set $X$. A semiorder (Luce 1956) is an irreflexive ${ }^{7}$ binary relation $P$ on $X$ that satisfies two additional properties.

1. $(x, y),(w, z) \in P$ implies $(x, z) \in P$ or $(w, y) \in P$.

2. $(x, y) \in P$ and $(y, z) \in P$ imply $(x, w) \in P$ or $(w, z) \in P$.

Given the irreflexivity of $P$, each of 1 or 2 imply that $P$ is also transitive. ${ }^{8}$ So a semiorder is a very special type of strict partial order. The appeal of semiorders is that they can be interpreted as a simple threshold model of (partial) rankings: on suitable domains, $P$ is a semiorder if and only if there exists a real-valued function $f$ on $X$ and a number $\sigma \geq 0$ such that $(x, y) \in P$ if and only if $f(x)>f(y)+\sigma$. Here $f(x)$ is the "value" of the alternative $x$ and $\sigma$ is the amount by which the value of one alternative $x$ must exceed the value of another alternative $y$ for $x$ to be declared superior to $y$. The fact that $\sigma$ is fixed makes this a very parsimonious model of binary preferences. ${ }^{9}$

Tversky (1969) essentially proposes a lexicographic procedure, which extends the use of semiorders, to make binary comparisons between alternatives in a set $X$. There exists an ordered sequence $f=\left(f_{1}, \ldots, f_{n}\right)$ of real valued functions on $X$ and a $\sigma>0$ such that $x$ is declared better than $y$ if and only if, for the first $i$ for which $\left|f_{i}(x)-f_{i}(y)\right|>\sigma$, we have $f_{i}(x)>f_{i}(y)+\sigma$. The idea is that the agent compares alternatives along several dimensions. As in our opening example, dimensions are ranked in order of importance, and a later dimension is considered only if all previous dimensions failed to discriminate between the two alternatives under consideration. In other words, the agent examines the dimensions lexicographically: as soon as a dimension $i$ is found for which one alternative $x$ is superior to another alternative $y$ by an amount exceeding the threshold $\sigma, x$ is declared better than $y$. When such an $i$ is found, no dimension $j$ that comes later in the order has any bearing, regardless of the size of the differences between the alternatives in these subsequent dimensions. That $\sigma$ is chosen to be the same for all $f_{i}$ is not a relevant issue, since even if we had different $\sigma_{i}$, the $f_{i}$ and $\sigma_{i}$ can always be rescaled so as to choose $\sigma_{i}=1$. Given $f$ and $\sigma$, this procedure can be used to generate a revealed preference relation $\succ_{(f, \sigma)}$ on pairs of alternatives. ${ }^{10}$

Suppose now that the agent wants to apply the procedure to produce a selection out of choice sets $S$ larger than the binary ones. There are several ways to do so, some of which are, however, problematic. One could, for example, start from the binary revealed preference relation and use either of the following two plausible methods.

- The choice from $S$ is the set of the maximal elements of $\succ_{(f, \sigma)}$.

\footnotetext{
${ }^{7}$ Irreflexivity: for all $x \in X,(x, x) \notin P$.

${ }^{8}$ Transitivity: for all $x, y, z \in X,(x, y) \in P,(y, z) \in P \Rightarrow(x, z) \in P$.

${ }^{9}$ In an interval order (Fishburn 1970), characterized by condition 1 alone, the threshold $\sigma$ is allowed to vary with the alternatives being compared, being a function $\sigma: X \rightarrow \mathbb{R}_{+}$. This makes for a much richer structure. See, e.g., Fishburn (1985).

${ }^{10}$ Rubinstein (1988) proposes a related but distinct procedure. This procedure is recently studied experimentally by Binmore et al. (2008).
} 
- The choice from $S$ is the top cycle (or the uncovered set) of $\succ_{(f, \sigma)}$ restricted to each $S .^{11}$

Unfortunately, the preference relation $\succ_{(f, \sigma)}$ may be cyclic: this "anomalous" feature was indeed the very point of Tversky's introduction of the procedure. So the first method above may not be well defined if a nonempty-valued choice function is desired. The second method above borrows the ideas of authors such as Ehlers and Sprumont (2008) and Lombardi (2008), who use weaker notions of maximization to produce choices out of nonstandard preferences formed of asymmetric and complete binary relations (tournaments). These methods would, for example, select the entire set $S=\left\{x_{1}, x_{2}, \ldots, x_{n}\right\}$ whenever $x_{1} \succ_{(f, \sigma)} x_{2} \succ_{(f, \sigma)} \cdots \succ_{(f, \sigma)} x_{n} \succ_{(f, \sigma)} x_{1}$.

Here we pursue a different natural way to extend Tversky's idea. The method we suggest is, on the one hand, more in line with the procedural (as opposed to maximizing) nature of Tversky's approach, and, on the other hand, can produce a unique selection even from the awkward cycles discussed above. The reason for these two features is that the method, unlike the others suggested, preserves and uses the information on the order in which the dimensions are considered.

We impose no arbitrary uniform bound on the number of dimensions that the agent is allowed to consider. Nevertheless, we insist that the procedure always halts in a finite number of steps in any choice situation.

Our proposed procedure works via a process of sequential elimination. Formally, let $\Sigma$ be a domain of choice sets, where each $S$ in $\Sigma$ is a nonempty subset of $X$. A choice function on $\Sigma$ is a function $c: \Sigma \rightarrow X$ such that $c(S) \in S$ for all $S \in \Sigma$. A choice set $S$ that has the form $S=\{x\}$ for some $x \in X$ is called trivial. A collection $\mathcal{C} \subseteq \Sigma$ of choice sets is trivial if each $S \in \mathcal{C}$ is trivial.

An ordered sequence $f=\left(f_{i}\right)_{i \in I}$, where $I$ is either an interval of numbers $\{1, \ldots, n\}$ or the entire set of natural numbers $\mathbb{N}$, together with a $\sigma>0$ is a lexicographic semiorder on $X$, denoted $\left(f_{1}, f_{2}, \ldots, \sigma\right)=\left(f_{i}, \sigma\right)_{i \in I}$. We abuse terminology and directly call each $f_{i}$ a semiorder, although strictly speaking $f_{i}$ is a numerical representation of it.

Given a choice set $S \subseteq X$ and a lexicographic semiorder $\left(f_{i}, \sigma\right)_{i \in I}$, define inductively the following "survivor sets" $M_{i}(S)$, for all $i>0$ :

$$
\begin{aligned}
& M_{0}(S)=S \\
& M_{i}(S)=\left\{s \in M_{i-1}(S) \mid \forall s^{\prime} \in M_{i-1}(S) f_{i}(s)+\sigma \geq f_{i}\left(s^{\prime}\right)\right\} .
\end{aligned}
$$

This sequence of sets captures the procedure the agent follows to arrive at a final selection from the choice set $S$ : at every round $i$, he looks for alternatives in the current survivor set $M_{i-1}(S)$ that are judged worse than some other alternative in $M_{i-1}(S)$ according to the Tversky procedure described before. He discards all such inferior alternatives (if any), generating the next survivor set $M_{i}(S)$ and so on.

\footnotetext{
${ }^{11}$ More precisely, let $P \mid S$ denote the restriction to $S$ of a complete asymmetric binary relation $P$ defined on $X$. (Completeness: For all $x, y \in X$, either $(x, y) \in P$ or $(y, x) \in P$. Asymmetry: For all $x, y \in X$, $(x, y) \in P \Rightarrow(y, x) \notin P$.) Let $(P \mid S)^{t}$ denote the transitive closure of $P \mid S$. The top cycle of $P$ in $S$ is the set of maximal elements of $(P \mid S)^{t}$ in $S$. Define the covering relation $C(P, S)$ of $P$ in $S$ by $(x, y) \in C(P, S)$ if and only if $x, y \in S$ and either $(x, y) \in P$ or there exists $z \in S$ such that $(x, z) \in P$ and $(z, y) \in P$. The uncovered set of $P$ in $S$ is the set of maximal elements of $C(P, S)$ in $S$.
} 
Definition 1. A choice function $c$ is a choice by lexicographic semiorder (cles) if there exists a lexicographic semiorder $\left(f_{i}, \sigma\right)_{i \in I}$ such that, for all $S \in \Sigma$, there is a $j \in I$ for which $\{c(S)\}=M_{j}(S)=M_{k}(S)$ for all $k \geq j$. In this case, we say that $\left(f_{i}, \sigma\right)_{i \in I}$ induces $c$.

That is, for a cles $c$, the iterative elimination procedure described before stops on any choice set $S$ after a finite number of steps, yielding precisely the alternative that $c$ picks in $S$. Note that, in spite of this property of "finite termination," there might not exist any fixed $j$ that works for all $S$. When such a $j$ exists, which means that $I$ can be chosen to be finite, we say that $c$ is a choice by finite lexicographic semiorder. ${ }^{12}$

\section{Basic semiorders}

A semiorder $f_{i}$ is basic if it ranges only in $\{-1,0,1\}$ and $\sigma=1$. A lexicographic semiorder $\left(f_{i}, \sigma\right)_{i \in I}$ is basic if each $f_{i}$ is basic. So with a basic lexicographic semiorder, the agent has only very limited power of discrimination. Essentially, on each dimension he can perform only a rough classification of alternatives into good ones (those $x$ for which $\left.f_{i}(x)=1\right)$, bad ones $\left(f_{i}(x)=-1\right)$, and neutral ones $\left(f_{i}(x)=0\right)$ : a good alternative beats a bad one (on the given dimension) and a neutral alternative neither beats a bad one nor is beaten by a good one.

A basic lexicographic semiorder can be denoted simply as $f=\left(f_{i}\right)_{i \in I}$. To emphasize that the survivor sets $M_{i}(S)$ are obtained from the basic lexicographic semiorder $f$, we write them as $M_{i}^{f}(S)$.

Example. Let $X=\{x, y, z\}$ and let $\Sigma=\{\{x, y\},\{y, z\},\{z, x\}, X\}$. Let $c(\{x, y\})=c(X)=x$, $c(\{y, z\})=y$, and $c(\{x, z\})=z$. This is a choice function by basic lexicographic semiorder. To see this, let $f_{1}(x)=0, f_{1}(y)=1, f_{1}(z)=-1, f_{2}(x)=1, f_{2}(y)=-1, f_{2}(z)=0$, $f_{3}(x)=-1, f_{3}(y)=1$, and $f_{3}(z)=1$. Observe how different (unique) choices from $X$ can be obtained by permuting the order of the $f_{i}$.

\section{Characterization}

Tversky thought that the model of binary choice by lexicographic semiorders, while useful to explain the anomaly of cyclical preferences, had a narrow scope otherwise. He writes (Tversky 1969, p. 40):

... despite its intuitive appeal, it is based on a noncompensatory principle that is likely to

be too restrictive in many contexts.

Following this logic, one might conjecture that the version with basic semiorders, with its minimal concession to discriminatory powers, is even more restrictive. We study this issue, highlighting the role of the domain of $c$.

We begin by observing that when restricted to binary choices (interpretable as possibly incomplete preferences) as in the original application, the cles model is, in fact,

\footnotetext{
${ }^{12}$ Aside from this twist, we could also call the model "semiorder sequentially rationalizable choice," following the terminology we initiated in Manzini and Mariotti (2007). However, we prefer to use the Tversky terminology, in recognition of the priority of his idea.
} 
completely unrestrictive provided that the set $X$ is not too large. We state this result separately because of its interest, although it is a particular case of the more general Proposition 2 below.

Proposition 1. Let $c$ be defined on a domain $\Sigma$ such that $S \in \Sigma$ implies $S=\{x, y\}$ for some distinct $x, y \in X$. Let $X$ be countable. Then there exists a basic lexicographic semiorder that induces $c$.

As noted, more generally all choice data satisfying a classical revealed preference axiom can be generated by a cles.

WARP. If $x=c(S), y \in S$, and $y=c(T)$ for some $S, T \in \Sigma$, then $x \notin T$.

Proposition 2. Let c satisfy WARP. Let X be countable. Then there exists a basic lexicographic semiorder that induces $c$.

Proof. Enumerate the elements in $X$ with a bijection $b$ from $\mathbb{N}$ (or an interval of $\mathbb{N}$ of cardinality equal to $|X|$ if $X$ is finite) to $X$. Define a basic lexicographic semiorder as follows. For all $x, y \in X$, let

$$
f_{b^{-1}(y)}(x)= \begin{cases}1 & \text { if } x=y \\ -1 & \text { if } \exists S \in \Sigma \text { such that } x \neq y=c(S) \text { and } x \in S \\ 0 & \text { otherwise. }\end{cases}
$$

Let $c(S)=x \neq y \in S$. WARP and the definition of $f$ imply that, for all $z \in X$ with $b^{-1}(z)<$ $b^{-1}(x), f_{b^{-1}(z)}(x)=0$ if $z=y$ and that $f_{b^{-1}(z)}(y) \leq 0$ whenever $f_{b^{-1}(z)}(x)=-1$. Therefore, $x \in M_{i}(S)$ for all $i<b^{-1}(x)$. And since $f_{b^{-1}(x)}(x)=1$ and $f_{b^{-1}(x)}(y)=-1$ for all $y \in S$ with $y \neq x,\{x\}=M_{b^{-1}(x)}(S)=M_{k}(S)$ for all $k \geq b^{-1}(x)$.

Because WARP does not imply SARP on general domains, ${ }^{13}$ a cles can explain cyclical patterns of strict revealed preference, generalizing Example.

To pinpoint the restrictions on behavior implied by the cles, we recall some definitions.

Definition 2. A choice function $c$ is sequentially rationalizable whenever there exists an ordered list $\left\{P_{i}\right\}_{i \in I}$ of asymmetric relations, with $P_{i} \subseteq X \times X$ for $i \in I$, such that, defining recursively,

$$
\begin{aligned}
& M_{0}^{*}(S)=S \\
& M_{i}^{*}(S)=\left\{x \in M_{i-1}^{*}(S) \mid \forall y \in M_{i-1}^{*}(S)(y, x) \notin P_{i}\right\}
\end{aligned}
$$

for all $S \in \Sigma$ there is a $j \in I$ such that

$$
\{c(S)\}=M_{j}^{*}(S)=M_{k}^{*}(S) \text { for all } k \geq j .
$$

\footnotetext{
${ }^{13}$ SARP says that the revealed preference relation $P_{c}$, given by $x P_{c} y \Leftrightarrow \exists S \in \Sigma: x=c(S), y \in S$, is acyclic.
} 
In that case we say that $\left\{P_{i}\right\}_{i \in I}$ sequentially rationalizes $c$. Each $P_{i}$ is a rationale. ${ }^{14}$

Two specializations of sequential rationalizability are the following.

Definition 3. A choice function is a rational shortlist method (RSM) if it is sequentially rationalizable with two rationales. A choice function is acyclic sequentially rationalizable if it is sequentially rationalizable by rationales that are acyclic.

Sequentially rationalizable choice functions and RSMs are defined in Manzini and Mariotti (2007). The restriction to acylic rationales for the finite case is studied by Apesteguia and Ballester (2010). Evidently, the cles model we are considering in this paper is a restriction of sequential rationalizability by constraining the rationales to be semiorders. Both acyclic and standard sequential rationalizability constitute at first sight a much more general model, because the rationales are not required to have any threshold structure and can thus apparently accommodate more sophisticated discriminations. But in fact, for arbitrary finite domains, the behaviors that can be generated by the lexicographic semiorder model and those that can be generated by the acyclic sequential rationalizability model are just the same. And we need look no further than basic semiorders to yield this equivalence.

On the other side of the coin, the restriction to finite domains is not merely a convenience for the inductive argument used in the proof, but it is necessary for the equivalence to hold. When the restriction is relaxed even marginally (by retaining the finiteness of each choice set but allowing for a countable number of choice sets), the model of acyclic sequential rationalizability suddenly appears to be far more general than the lexicographic semiorder model: even only two acyclic rationales suffice to produce behaviors that cannot be induced by any basic lexicographic semiorder. And increasing the discriminatory ability of the agent is to no avail: the basic restriction is inessential for this result.

These assertions are made precise in the next two results. In the case of the domain consisting of all finite subsets, the first result can also be derived from Theorem B.1 in Apesteguia and Ballester (2010), as we explain below. We present here a different method of proof that highlights the importance of the domain and is instructive in this respect.

Proposition 3. Let $X$ be finite. Then a choice function $c$ is acyclic sequentially rationalizable if and only if it is induced by a basic lexicographic semiorder.

Proof. A semiorder is an acyclic rationale, so it suffices to prove the "only if" part of the statement. Given acyclic rationales $\left(P_{1}, \ldots, P_{K}\right)$, recall Definition 2 of survivor sets $M_{i}^{*}(S)$. We show that, for any domain $\Sigma$, there exists a basic lexicographic semiorder

\footnotetext{
${ }^{14}$ This definition slightly extends the one we originally gave (Manzini and Mariotti 2007), and has the same format of the definition of a lexicographic semiorder. In the original definition we considered a finite ordered list $P_{1}, \ldots, P_{K}$ of asymmetric relation with the $\{c(S)\}=M_{K}^{*}(S)$ for all $S \in \Sigma$. While still imposing finite termination on each choice set, the current definition disposes with the assumption that there exists a uniform bound $K$ on the number of rationales needed to rationalize a given choice function.
} 
$f=\left(f_{i}\right)_{i \in I}$ such that, for all $S \in \Sigma$, there is a $j \in I$ such that $M_{K}^{*}(S)=M_{j}^{f}(S)=M_{k}^{f}(S)$ for all $k \geq j$. This proves the assertion in the statement.

The proof is by induction on the sum of the cardinalities of the sets $S$ in $\Sigma$, which we denote by $n(\Sigma)=\sum_{S \in \Sigma}|S|$. If $n(\Sigma)=1$, the claim is obviously true. Take now $n(\Sigma)>1$. If $\Sigma$ is trivial, then the claim is also obviously true, so assume $\Sigma$ is not trivial, and without loss of generality assume in addition that $P_{1}$ is nonempty on some $S \in \Sigma$ (otherwise just exclude $P_{1}$ and renumber the remaining $P_{i}$ ). By the acyclicity of $P_{1}$ and the finiteness of $X$, there exist $S \in \Sigma$ and $x, y \in S$ such that $(x, y) \in P_{1}$ and $(y, z) \notin P_{1}$ for all $z \in \bigcup_{S \in \Sigma} S$ with $y, z \in T$ for some $T \in \Sigma$ (in words, $y$ is $P_{1}$-dominated in some choice set and it does not $P_{1}$-dominate any element that appears together with $y$ in any choice set). Fix those $x$ and $y$, and define

$$
\begin{aligned}
\Sigma^{\prime}= & \{S \mid\{x, y\} \nsubseteq S \in \Sigma\} \\
& \cup\{S \mid S=T \backslash\{y\} \text { for some } T \in \Sigma \text { such that }\{x, y\} \subseteq T\} .
\end{aligned}
$$

Because a $T$ as in the right-hand member of the union above exists by construction, $n\left(\Sigma^{\prime}\right)<n(\Sigma)$. So by the inductive hypothesis, there exists a basic lexicographic semiorder $f=\left(f_{i}\right)_{i \in I}$ such that, for all $S \in \Sigma^{\prime}$, there is a $j \in I$ such that $M_{K}^{*}(S)=M_{j}^{f}(S)=$ $M_{k}^{f}(S)$ for all $k \geq j$. Now consider the basic lexicographic semiorder $g=\left(g_{i}\right)_{i \in I^{\prime}}$ defined by

$$
\begin{aligned}
g_{i} & =f_{i-1} \quad \text { for all } i>1 \\
g_{1}(x)=1, \quad g_{1}(y) & =-1 \quad \text { and } \quad g_{1}(z)=0 \text { for all } z \neq x, y .
\end{aligned}
$$

Thus, for all $S \in \Sigma$ such that $\{x, y\} \subseteq S, M_{1}^{g}(S)=S \backslash\{y\} \in \Sigma^{\prime}$ and consequently $M_{K}^{*}(S \backslash$ $\{y\})=M_{j+1}^{g}(S)=M_{k}^{g}(S)$ for all $k \geq j+1$ (this follows by the second line of the displayed definition of $g$ and the fact that $M_{K}^{*}(S \backslash\{y\})=M_{j}^{f}(S \backslash\{y\})=M_{k}^{f}(S \backslash\{y\})$ for all $\left.k \geq j\right)$. Moreover, clearly for all $S \in \Sigma$ such that $\{x, y\} \subseteq S, M_{K}^{*}(S)=M_{K}^{*}(S \backslash\{y\})$. Therefore, for all $S \in \Sigma, M_{K}^{*}(S)=M_{K}^{*}(S \backslash\{y\})=M_{j+1}^{g}(S)=M_{k}^{g}(S)$ for all $k \geq j+1$.

Proposition 4. There exist rational shortlist methods using acyclic rationales that are not induced by any lexicographic semiorder.

Proof. Let $X=\{1,2, \ldots\}$, let $\Sigma$ be the collection of finite subsets of $X$, and let $c$ be uniquely defined as the RSM rationalized by the two acyclic rationales

$$
P_{1}=\{(i, i+1) \mid i \in X\}
$$

and

$$
P_{2}=\{(j, i) \mid j>i+1\} .
$$

We show that $c$ is not induced by any lexicographic semiorder. By way of contradiction, suppose that $\left(f_{\alpha}, \sigma\right)_{\alpha \in I}$ is a lexicographic semiorder that induces $c$. Let $i, j \in X$ be such that $f_{1}(j)>f_{1}(i)+\sigma$. Such an $i$ and $j$ exists without loss of generality, possibly by renumbering the $f_{\alpha}$ so that $f_{1}$ is the first $f_{\alpha}$ for which $f_{1}\left(k^{\prime}\right)>f_{1}(k)+\sigma$ for 
some $k, k^{\prime} \in X$. Also note that $i \neq 1$, since the application of the rationales yields $c(\{1,2, \ldots, l\})=1$ for all $l \in X$. It must be that $j=i-1$ (that is, $i$ is eliminated by $i-1$ in the first step in any set that contains both of them). Otherwise suppose first that $j>i$. Then $c(\{i, i+1, i+2, \ldots, j\})=i$ is contradicted by $i \notin M_{1}(\{i, i+1, i+2, \ldots, j\})$. Alternatively, suppose that $j<i-1$. Then $c(\{j, i\})=i$ is contradicted by $i \notin M_{1}(\{j, i\})$.

Thus, $f_{1}(i-1)>f_{1}(i)+\sigma$. Since $c(\{i-1, i+1\})=i+1$, it must be that, letting $n$ be the first $\alpha$ for which $M_{\alpha}(\{i-1, i+1\}) \neq\{i-1, i+1\}$, we have $f_{n}(i+1)>f_{n}(i-1)+\sigma$. Applying this fact to $S=\{i-1, i, i+1\}$, we have that if $n=1$, then $M_{1}(S)=\{i+1\}$, contradicting $c(S)=i-1$. If instead $n>1$, then either $f_{1}(i)>f_{1}(i+1)+\sigma$, in which case we also have $f_{1}(i-1)>f_{1}(i+1)+\sigma$, contradicting $c(\{i-1, i+1\})=i+1$, or this is not the case, so that $c(S)=c\left(M_{1}(S)\right)=c(\{i-1, i+1\})=i+1$. In both cases we have a contradiction with $c(S)=i-1 .^{15}$

Some observations are in order. Apesteguia and Ballester (2010) define a simple rationale $P$ as a relation of the type $P=\{(x, y)\}$ for some $x$ and $y$ in $X$. That is, a simple rationale relates only one pair of alternatives. Our notion of 'basic' refers instead to the number of discriminations the agent is able to make, rather than to the number of pairs ranked by the relation (which may be high). However, Apesteguia and Ballester (2010) show that, for the case of the domain consisting of all subsets of a finite set $X$, sequential rationalizability with acyclic rationales is equivalent to sequential rationalizability with simple rationales. The ranking made by a simple rationale $P=\{(x, y)\}$ can be expressed with a basic semiorder (though not vice-versa), by setting $f(x)=1, f(y)=-1$, and $f(z)=0$ for all other $z$. Therefore, as observed above, Proposition 3 can be derived by their result in the case of full domain.

While a simple rationale can be expressed by means of a single semiorder, there is no upper bound to the number of simple rationales needed to express a basic semiorder. For example, the rationale $P=\{(x, y) \mid y \in X \backslash\{x\}\}$, for a fixed $x$, is a single basic semiorder for any $n$, which is nevertheless decomposed into $(n-1)$ distinct simple rationales. $^{16}$

Proposition 4 shows that the domain restriction $|X|<\infty$ of Theorem B.1 of Apesteguia and Ballester (2010) is necessary. Their result establishes that on the domain of all nonempty subsets of a finite set $X$, the only crucial distinction is between the asymmetry and the acyclicity (a strengthening of asymmetry) of the rationales: further strengthening acyclicity to transitivity, for example, produces no further behavioral restriction. Proposition 4 shows that on larger domains, the move from acyclicity to transitivity (semiorders) crosses another important threshold: the transitivity of the agent's discriminatory power alone suffices to rule out behaviors allowed by acyclic rationales. This remains true no matter how limited that power is.

\footnotetext{
${ }^{15}$ Observe that it is at this step of the proof that the domain assumption bites, for $\{i-1, i, i+1\}$ and $\{i-1, i+1\}$ might not be well defined if we did not have the entire integer set at our disposal.

${ }^{16}$ In recent work, Mandler (2009) studies in detail the general issue of the minimum number of rationales needed to express a given arbitrary preference relation (interpretable as the base relation of a choice function) using the procedure of sequential rationalizability. His main result is that a "rational agent" (an agent with complete and transitive preferences) never needs more, and sometimes needs fewer, rationales than a nonrational agent.
} 


\section{1 "Revealed preference" characterization}

How can an external observer establish whether a set of choice data (i.e., a choice function c) can be generated by the procedure we propose? The key to answering this question is to consider the behavior of $c$ over restricted domains of choice, as well as on the domain $\Sigma$ of the definition of $c$. The method we suggest can be viewed as an extension of techniques used in standard analysis of rationalizability of choice functions on special domains. For example, in recent work, Kalandrakis (2010) studies the rationalizability of a set of binary voting choices on Euclidean policy space by means of a quasiconcave utility function. He identifies rationalizability conditions with the following format: for every subdomain of choice $\mathcal{C}$, there exists an "extreme alternative" (i.e., not obtainable as a convex combination of other alternatives) $x$ such that $x$ is never chosen from choice sets in the collection $\mathcal{C}$. The interpretation is that $x$ is a least preferred alternative among those appearing in the choice problems in $\mathcal{C}$. This permits the ultimate construction of a (quasiconcave) utility function.

Let us see how analogous ideas can work in our setting. Because our model does not involve the simple maximization of preferences, we cannot hope to identify "least preferred" alternatives. But if the agent were really using our lexicographic procedure, in any subdomain $\mathcal{C}$, we should at least be able to identify alternatives $x$ and $y$ such that $x$ makes $y$ "C-irrelevant": namely, if $x$ and $y$ belong to some $S$ in $\mathcal{C}$, removing $y$ from $S$ has no effect on the final choice from $S$ (so that, in particular, $y$ is never chosen if $x$ is available). This alternative $y$ is simply one of the alternatives the agent would eliminate with the first semiorder that he applies on $\mathcal{C}$, say $f_{\mathcal{C}}$, and $x$ is an alternative with $f_{\mathcal{C}}(x)>f_{\mathcal{C}}(y)+\sigma$. In other words, given any $\mathcal{C}$, an agent following our procedure should always implicitly indicate at least one pair $(x, y)$ where $x$ makes $y \mathcal{C}$-irrelevant.

To illustrate, take any choice function $c$ (defined on a possibly large domain $\Sigma$ ) for which $c(\{x, y\})=c(\{x, z\})=x, c(\{x, y, z\})=y$. Consider the subdomain $\mathcal{C}=\{\{x, y\}$, $\{x, z\},\{x, y, z\}\}$. Because the agent has chosen $x$ both from $\{x, y\}$ and $\{x, z\}$, so that $x$ is not made $\mathcal{C}$-irrelevant by either of the other alternatives, the agent is "indicating" that, even if he were using a lexicographic heuristic, the first rationale that is active on $\mathcal{C}$ would not eliminate $x$. Similarly, because $c(\{x, y, z\}) \neq c(\{x, z\})$ and $c(\{x, y, z\}) \neq c(\{x, y\})$, so that neither $y$ nor $z$ are $\mathcal{C}$-irrelevant, the agent is also indicating that the first active rationale would not eliminate $y$ or $z$. Thus, no alternative can be eliminated and we can conclude that the agent cannot possibly be choosing according to our lexicographic procedure.

The remarkable thing is that the following axiom, which formalizes this intuition, is not only necessary, but turns out to embody all the observable implications of the model.

Reducibility. For every nonempty $\mathcal{C} \subseteq \Sigma$, there exist $S \in \mathcal{C}$ and $x, y \in S$ such that, for all $T \in \mathcal{C}$,

$$
(T \backslash\{y\}) \in \mathcal{C}, \quad x \in T \Rightarrow c(T)=c(T \backslash\{y\}) .
$$

A choice function that satisfies Reducibility is called reducible. 
If $x$ makes $y \mathcal{C}$-irrelevant, we cannot identify from choice data alone why this is the case. It could simply be that $x$ is better than $y$ (e.g., superior by any criterion). But it could also be that $x$ is pizza, $y$ is steak tartare, and you simply ignore steak tartare in any restaurant that also offers pizza (although you may or may not choose pizza). Here, pizza might be a negative signal about the kitchen's sophistication, so that you are induced to ignore sophisticated items on the menu, even if you may end up not choosing the signal item itself. $^{17}$

Obviously, one extreme way of satisfying Reducibility is the existence of a "best" alternative. If $c$ is a choice function that maximizes an ordinary strict preference relation, an alternative that is chosen from an $S$ in $\mathcal{C}$ trivially makes $\mathcal{C}$-irrelevant any alternative that is not chosen from $S$. In fact, in standard theory "irrelevant" is essentially synonymous with "unchosen." Therefore, $c$ is reducible in the standard case.

Reducibility relaxes the standard requirement that all rejected alternatives need to be made $\mathcal{C}$-irrelevant on all $\mathcal{C}$ (via the single preference relation) by the "best" (chosen) alternative, and it does so in two ways. First, some rejected alternatives may not be made $\mathcal{C}$-irrelevant. And, second, an alternative may be made $\mathcal{C}$-irrelevant by some other alternative that itself is not chosen. In other words, Reducibility requires just a bare skeleton of preference to survive.

An example of a reducible nonstandard choice function is the three-cycle of choice: $X=\{x, y, z\}, c(X)=c(\{x, y\})=x, c(\{y, z\})=y, c(\{x, z\})=z$. Here $y$ makes $z \mathcal{C}$-irrelevant when either $X$ or $\{y, z\}$ is in $\mathcal{C}$, and Reducibility is satisfied vacuously otherwise. Observe that the choice from the grand set does not make either $y$ or $z \mathcal{C}$-irrelevant for $\mathcal{C}$ coinciding with the full domain.

On the contrary, the choice function $c$ in the proof of Proposition 4 (where $c$ is sequentially rationalizable but not cles) is not reducible. In that example, Reducibility fails on the collection $\mathcal{C}=\Sigma$. To see this, observe that no $i$ can make $i+1 \mathcal{C}$-irrelevant, since we would have the contradiction $c(\{i, i+1, i+2\})=i \neq i+2=c(\{i, i+2\})$. Also, $i+1$ cannot make $i \mathcal{C}$-irrelevant for $c(\{i, i+1\})=i$. Moreover, no two nonadjacent alternatives $i$ and $j$ with $j>i+1$ are suitable either. It cannot be that $j$ makes $i \mathcal{C}$-irrelevant since $c(\{j, j-1, \ldots, i+2, i\})=i$, and it cannot be that $i$ makes $j \mathcal{C}$-irrelevant since $c(\{i, j\})=j$. This reasoning also highlights the role that infinite domains play in separating lexicographic semiorders from sequentially rationalizable choice.

Reducibility is easily seen to be a weakening of a standard contraction consistency axiom. Consider the following formulation.

Independence of Irrelevant Alternatives (IIA). Let $\mathcal{C} \subseteq \Sigma$. Then $c(S)=c(S \backslash\{y\})$ for all $y \in S \backslash\{c(S)\}$ for all $S \in \mathcal{C}$ such that $S \backslash\{y\} \in \Sigma$.

Now consider the following weakening (where we highlight in boldface the additional conditions).

\footnotetext{
${ }^{17}$ In this example, pizza plays a symmetric role to that of frog legs in the celebrated example by Luce and Raiffa (1957) (a decision maker chooses steak when frog legs are on the menu and salmon when they are not). In Luce and Raiffa's example, frog legs are a positive signal about the quality of the restaurant, so that the decision maker is induced by the presence of frog legs on the menu to choose a high quality item, even if not frog legs themselves.
} 
Reducibility (Restated). Let $\mathcal{C} \subseteq \Sigma$. Then for some $x \in X, c(S)=c(S \backslash\{y\})$ for some $y \in S \backslash\{c(S)\}$ for all $S \in \mathcal{C}$ such that $S \backslash\{y\} \in \Sigma$ and $S \ni x$.

While standard IIA requires the choice to be unchanged if any unchosen alternative is removed from any set, Reducibility requires this to hold only for some alternative and for some sets (those containing $x$ ). Because IIA is so strong, the fact that if it holds, it must hold on the entire domain $\Sigma$ as well as on any subcollection $\mathcal{C}$, usually does not need to be made explicit.

Below we establish that Reducibility identifies all the observable implications of the lexicographic semiorder procedure and that basic lexicographic semiorders cover exactly the same ground as general lexicographic semiorders.

Theorem 1. Let $X$ be finite. Let $c$ be a choice function defined on the domain $\Sigma$ of all finite subsets of $X$. Then the following statements are equivalent.

(i) The function $c$ is a choice by lexicographic semiorder.

(ii) The function c is reducible.

(iii) The function c is a choice by basic lexicographic semiorder.

Proof. (i) $\Rightarrow$ (ii). Let $c$ be induced by the lexicographic semiorder $\left(f_{i}, \sigma\right)_{i \in I}$ and let $\mathcal{C} \subseteq \Sigma$ be any nontrivial collection of choice sets. Let

$$
j=\min \left\{i \mid M_{i}(S) \neq S \text { for some } S \in \mathcal{C}\right\}
$$

( $j$ is well defined because of the single valuedness of $c$ ). ${ }^{18}$

Let $T \in \mathcal{C}$ be such that $M_{j}(T) \neq T$. Fix $x, y \in T$ such that $f_{j}(x)>f_{j}(y)+\sigma$. For any $S \in \mathcal{C}$, either $\{x, y\} \nsubseteq S$, in which case Reducibility holds vacuously, or $\{x, y\} \subseteq S$. In this latter case (which holds at least for $S=T$ ), for any $z \in S$, if $f_{j}(y)>f_{j}(z)+\sigma$, then also $f_{j}(x)>f_{j}(z)+\sigma$. Therefore $M_{j}(S)=M_{j}(S \backslash\{y\})$, implying $c(S)=c(S \backslash\{y\})$.

(ii) $\Rightarrow$ (iii). Let $c$ be a reducible choice function on $\Sigma$. We first provide an algorithm to construct a basic lexicographic semiorder for any choice function, then show that this semiorder induces $c$.

The algorithm proceeds by recursively defining a sequence of collections $\left\{\mathcal{C}_{i}\right\}_{i \in I}$ and an associated sequence of pairs $\left\{x_{i}, y_{i}\right\}_{i \in I}$, where $I$ is the interval $\{0,1, \ldots, n\}$ for some $n$. Let $\mathcal{C}_{0}=\Sigma$ and let $x_{0}, y_{0} \in X$ be any two alternatives such that, for all $S \in \mathcal{C}_{0}, x_{0}, y_{0} \in S \Rightarrow$ $c(S)=c\left(S \backslash\left\{y_{0}\right\}\right)$ (alternatives such as $x_{0}$ and $y_{0}$ exist by Reducibility, and $S \backslash\left\{y_{0}\right\} \in \Sigma$ by assumption). For $0<i$, define recursively $x_{i}, y_{i} \in X$ as any two alternatives such that $\left(x_{i}, y_{i}\right) \neq\left(x_{j}, y_{j}\right)$ for all $j<i$, and

$$
\text { for all } S \in \bigcap_{j<i} \mathcal{C}_{j}: \quad x_{i}, y_{i} \in S \Rightarrow c(S)=c\left(S \backslash\left\{y_{i}\right\}\right)
$$

\footnotetext{
${ }^{18}$ For choice correspondences one would change the qualifier "not all $S$ in $\mathcal{C}$ are singletons" with "not all of them are such that $c(S)=S$."
} 
and

$$
\mathcal{C}_{i}=\bigcap_{j<i} \mathcal{C}_{j} \backslash\left\{S \in \bigcap_{j<i} \mathcal{C}_{j} \mid\left\{x_{i}, y_{i}\right\} \subseteq S\right\} .
$$

For all $i$, let $f_{i}\left(x_{i}\right)=1, f_{i}\left(y_{i}\right)=-1, f_{i}(z)=0$ for all $z \in X \backslash\left\{x_{i}, y_{i}\right\}$, and $\sigma=1$. Because $X$ is finite, for any $i$, unless $S \in \mathcal{C}_{i+1} \Rightarrow|S|=1$ (i.e., unless $\mathcal{C}_{i}$ is a trivial collection), it is true by Reducibility that $\mathcal{C}_{i} \neq \mathcal{C}_{i+1}$. Therefore, $S \in \bigcap_{i \in I} \mathcal{C}_{i} \Rightarrow|S|=1$.

This defines a basic lexicographic semiorder $f=\left(f_{i}\right)_{i \in I}$. As we show below, $f$ induces $c$. Recall the definition of the survivor sets $M_{i}(S)$.

Fix $S \in \Sigma$. Suppose by induction that $c(S) \in M_{i}(S)$. It must be that $M_{i}(S) \in \mathcal{C}_{i}$. Otherwise, there would exist $k \leq i$ such that $f_{k}\left(x_{k}\right)=1, f_{k}\left(y_{k}\right)=-1$, and $\left\{x_{k}, y_{k}\right\} \subseteq M_{i}(S) \in \mathcal{C}_{k}$, contradicting the definition of $M_{i}(S)$. If also $M_{i}(S) \in \mathcal{C}_{i+1}$, then $\left\{x_{i+1}, y_{i+1}\right\} \nsubseteq M_{i}(S)$ and so we have immediately $c(S) \in M_{i+1}(S)$. If $M_{i}(S) \notin \mathcal{C}_{i+1}$, then (since $\left.M_{i}(S) \in \mathcal{C}_{i}\right)$ it must be $\left\{x_{i+1}, y_{i+1}\right\} \subseteq S$. It cannot be $y_{i+1}=c(S)$ since, by construction of the sequence $\left\{x_{i}, y_{i}\right\}_{i \in I}$, $c(S)=c\left(S \backslash\left\{y_{1}\right\}\right)=\cdots=c\left(S \backslash\left\{y_{1}, \ldots, y_{i+1}\right\}\right)$. Therefore, $c(S) \in M_{i+1}(S)$.

We now show that for all $s \in S \backslash\{c(S)\}$, there exists a $k$ such that $s \notin M_{k}(S)$. If not, let $\bigcap_{i \in I} M_{i}(S)=T$ and let $s \in T$. The definition of $T$ implies that, for all $i \in I$, $\left\{x_{i}, y_{i}\right\} \nsubseteq T$ (otherwise $x_{i}, y_{i} \in M_{i}(S)$, which is impossible by construction since $f_{i}\left(x_{i}\right)=1$ and $\left.f_{i}\left(y_{i}\right)=-1\right)$. Therefore, $T \in \bigcap_{i \in I} \mathcal{C}_{i}$. But this is a contradiction with $c(S) \neq s \in T$ and $c(S) \in T$, since, as observed before, $T \in \bigcap_{i \in I} \mathcal{C}_{i}$ implies $|T|=1$.

(iii) $\Rightarrow$ (i). Trivial.

Remark 1. The proof of Theorem 1 implies, in fact, an even more general result. The only feature of a lexicographic semiorder that we use in the proof is transitivity. Therefore, the same characterization would hold even for procedures that use generic partial orders at each stage of elimination.

\subsection{Sequentially rationalizable choice}

Theorem 1 can be used together with Proposition 3 to provide a characterization of acyclic sequential rationalizability:

Corollary 1. Let $X$ be finite and let $\Sigma$ be the set of all nonempty subsets of $X$. Then a choice function on $\Sigma$ is acyclic sequentially rationalizable if and only if it is reducible.

In short, then, while acyclic sequential rationalizability and lexicographic semiorders coincide on finite sets, they are nested for choice functions defined over more general domains (see Proposition 4). This observation prompts the natural question, What types of behavior can be explained by the sequential rationalizability model but not by the lexicographic semiorder model? To this aim, we introduce a weakening of Reducibility.

Weak Reducibility. For every nonempty $\mathcal{C} \subseteq \Sigma$, there exists $S \in \mathcal{C}$ and a collection of pairs $\left\{x_{i}, y_{i}\right\}_{i=1,2, \ldots}$, with $x_{i}, y_{i} \in S$ for all $i$, such that, for all $T \in \mathcal{C}$,

$$
T \backslash \bigcup_{i: x_{i} \in T}\left\{y_{i}\right\} \in \mathcal{C} \Rightarrow c(T)=c\left(T \backslash \bigcup_{i: x_{i} \in T}\left\{y_{i}\right\}\right) .
$$


A choice function that satisfies Weak Reducibility is called weakly reducible.

The only difference between Reducibility and Weak Reducibility is that in the latter, the single pair $(x, y)$ is replaced by a collection $\left\{x_{i}, y_{i}\right\}_{i=1,2, \ldots}$ of pairs. In other words, compared to a reducible choice function, a choice function that is only weakly reducible is such that some alternatives that are not individually $\mathcal{C}$-irrelevant (the removal of any one of those alternatives does affect choice) may nevertheless be "collectively" $\mathcal{C}$ irrelevant (their collective removal from a choice set has no relevance for choice).

We show that the choice functions that are sequentially rationalizable but not cles are exactly those that are only weakly reducible but not reducible.

Theorem 2. Let $X$ be finite. Let $c$ be a choice function defined on the domain $\Sigma$ of all finite subsets of $X$. Then $c$ is sequentially rationalizable if and only if it is weakly reducible.

Proof. Necessity. Let $c$ be sequentially rationalizable with rationales $\left\{P_{i}\right\}_{i \in I}$ and let $\mathcal{C} \subseteq \Sigma$. Let

$$
j=\min \left\{i \mid M_{i}^{*}(S) \neq S \text { for some } S \in \mathcal{C}\right\} .
$$

Let $A=\left\{(x, y) \mid x, y \in S\right.$ for some $S \in \mathcal{C}$ and $\left.(x, y) \in P_{j}\right\} ; A$ is nonempty by the definition of $j$. Enumerate the pairs in $A$ to obtain $\left\{x_{i}, y_{i}\right\}_{i \in J}$, where $J$ is the finite interval $\{1,2, \ldots, n\}$ for some $n$. Let $\bar{j}(S)=\min \left\{j \mid M_{j}(S)=M_{k}(S)\right.$ for all $\left.k \geq j\right\}$. Note that $\bar{j}$ is well defined since $c$ is sequentially rationalizable. It follows straightforwardly that $M_{\bar{j}(S)}^{*}(S)=M_{\bar{j}(S)}^{*}\left(S \backslash \bigcup_{i: x_{i} \in S}\left\{y_{i}\right\}\right)$ for all $S \in \mathcal{C}$. The sequential rationalizability of $c$ thus implies that $c(S)=c\left(S \backslash \bigcup_{i: x_{i} \in S}\left\{y_{i}\right\}\right)$.

Sufficiency. Let $c$ be weakly reducible. We construct the rationales explicitly. Let $\mathcal{C}_{0}=\Sigma$ and define recursively

$$
\begin{aligned}
P_{i}= & \left\{\left(x_{j i}, y_{j i}\right)\right\}_{j=1, \ldots, n(i)} \\
& \text { where }\left\{x_{j i}, y_{j i}\right\}_{j=1, \ldots, n(i)} \text { is any collection of pairs such that } \\
c(S)= & c\left(S \backslash \bigcup_{j: x_{j i} \in S}\left\{y_{j i}\right\}\right) \forall S \in \mathcal{C}_{i-1} \\
\mathcal{C}_{i}= & \left\{S \in \mathcal{C}_{i-1} \mid S=M_{i}^{*}(T) \text { for some } T \in \mathcal{C}_{i-1}\right\} .
\end{aligned}
$$

The $P_{i}$ are well defined by Weak Reducibility. Similarly to the proof of Theorem 1, unless $S \in \mathcal{C}_{i+1} \Rightarrow|S|=1$ (i.e., unless $\mathcal{C}_{i}$ is a trivial collection), it is true by Weak Reducibility that $\mathcal{C}_{i} \neq \mathcal{C}_{i+1}$. Therefore, $S \in \bigcap_{i \in I} \mathcal{C}_{i} \Rightarrow|S|=1$. We show that $\left\{P_{i}\right\}_{i \in I}$, where $I$ is the interval $\{1,2, \ldots, n\}$ for some $n$, sequentially rationalize $c$.

Let $x=c(S)$. Whenever $S \in \mathcal{C}_{i-1}$ for some $i$, it cannot be $(y, x)=P_{i}$, since $c(S) \neq c(S \backslash$ $(\{x\} \cup A)$ ) for any $A \subseteq X$, contradicting the definition of $P_{i}$. This implies that $x \in M_{i}^{*}(S)$ for all $i$.

We now show that for all $y \in S \backslash\{c(S)\}$ there exists a $k$ such that $y \notin M_{k}(S)$. If not, let $\bigcap_{i \in I} M_{i}(S)=T$ and let $y \in T$. The definition of $T$ implies that, for all $i \in I$, $\left\{x_{j i}, y_{j i}\right\}_{j=1, \ldots, n(i)} \nsubseteq T$ (otherwise $x_{j i}, y_{j i} \in M_{i}(S)$, which is impossible by construction 
since $\left.\left(x_{j i}, y_{j i}\right) \in P_{i}\right)$. Therefore, $T \in \bigcap_{i \in I} \mathcal{C}_{i}$. But this is a contradiction to $c(S) \neq y \in T$ and $c(S) \in T$, since, as observed before, $T \in \bigcap_{i \in I} \mathcal{C}_{i}$ implies $|T|=1$.

Theorems 1 and 2 are interesting in themselves, as Manzini and Mariotti (2007) left the characterization of sequential rationalizability an open problem.

Apesteguia and Ballester (2010) pioneered a solution to that problem, in so doing offering key insights. Their characterization of acyclic sequential rationalizability is in terms of a condition called Independence of One Irrelevant Alternative (IOIA). To quickly sketch that condition, we need to define some auxiliary terms. A binary selector is a function $f$ that associates to every feasible set $S$, including at least two alternatives to a binary feasible set in $S$. A binary selector $f$ that satisfies certain consistency properties $^{19}$ is called consistent. Then IOIA requires that $c(S)=c(S \backslash(f(S) \backslash\{c(f(S))\}))$ for some consistent binary selector. While this condition may appear involved, its broad logic is simple, as it essentially imposes a two-stage structure on the choice function $c$. This is convenient because it reduces the problem of detecting an arbitrarily long sequential structure on $c$ to that of detecting a far simpler construction. Thus, IOIA and Reducibility, which by our results and those of Apesteguia and Ballester (2010) are equivalent conditions in the finite case, highlight different aspects of sequential rationalizability. In both cases, the key idea is to somehow identify the sequence by which eliminations are made. Roughly speaking, both axioms try to identify the next step in the elimination process: IOIA's consistent binary selector tells which pair gets compared next out of any given set; Reducibility tells which pair gets compared next out of a given set of sets. ${ }^{20}$

As we already noted, in the case of finite domains, what really matters is the move from asymmetric to acyclic rationales-further strengthening the discriminatory power of each criterion to transitivity implies no additional restrictions for observed behavior. However, when moving to larger domains, this conclusion no longer holds. More precisely, for the case of countable domains, we already saw (Proposition 4) that there are RSMs with acyclic rationales that are not cles; in addition, the proof of Proposition 4 can be easily modified to show that there are choice functions that are RSM's with acyclic rationales but that cannot be induced by any sequence of transitive rationales. ${ }^{21}$ Furthermore, it can be shown that there are choice functions that are sequentially rationalizable

\footnotetext{
${ }^{19}$ We refer the reader to Apesteguia and Ballester (2010) for a precise statement of the definition, which requires substantially more notation extraneous to the purposes of this paper.

${ }^{20} \mathrm{We}$ are grateful to Bart Lipman for suggesting this interpretation of the relation between the two conditions. An explicit proof of the equivalence between the two conditions in the finite case, as well as an example of their nonequivalence in the infinite case, is available from the authors upon request.

${ }^{21}$ Take the $c$ function induced by the RSM in the proof of Proposition 4 and suppose, by way of contradiction, that $Q_{1}, \ldots, Q_{K}$ is a list of transitive rationales that rationalizes $c$. For any two alternatives $i$ and $j$, let $k(i, j)=\min \left\{k \mid(i, j) \in Q_{k}\right.$ or $\left.(j, i) \in Q_{k}\right\}$. Fix some $i$ and $j$ such that $(i, j) \in Q_{1}$, and observe that $j \neq 1$, for otherwise $c(\{1,2, \ldots, l\})=1$ for all $l \in X$ could not be retrieved. Moreover, it must be that $i=j-1$ : if either $i<j-1$ or $i=j+1$, then the choice $c(\{i, j\})=j$ could not be retrieved, while if $i>j+1$, then $c(\{j, j+1, \ldots, i\})=j$ could not be retrieved. So $(i, i+1) \in Q_{1}$, and since $Q_{1}$ is transitive, it must be $(i+2, i) \notin Q_{1}$, for otherwise $(i+2, i+1) \in Q_{1}$ and $c(\{i+1, i+2\})=i+1$ could not be retrieved. But then if $(i+1, i+2) \in Q_{1}$, we cannot retrieve $c(\{i, i+2\})=i+2$, since by transitivity $(i, i+2) \in Q_{1}$, while if $(i+1, i+2) \notin Q_{1}$ we cannot retrieve $c(\{i, i+1, i+2\})=i$ (observe that $(i+2, i) \in Q_{k(i, i+2)}$ since $c(\{i, i+2\})=i+2)$.
} 
by means of transitive rationales, but not by a lexicographic semiorder. ${ }^{22}$ In sum, for domains with countable $X$, we have that sequential rationalizability by means of asymmetric rationales is more permissive than when acyclic rationales are used, which is, in turn, more permissive than in the case when transitive rationales are required, and, in turn, cles are even more restrictive in terms of observable choice behavior.

The challenge ahead is to provide characterizations in this vein for very general domains, including, for example, those of standard consumer theory. This remains an open question. Of course, in domains that are not finite, if one insists on finite termination on each set, the procedure underlying choice by lexicographic semiorder only leads to a unique selection only if (at least some of) the criteria are capable of eliminating an infinite number of alternatives. For those domains, one may have to accept, in general, that a choice correspondence, instead of a choice function, is the appropriate primitive. In particular, imagine that the procedure studied so far describes only a preselection of suitable alternatives (a shortlist), while a final unique choice might be delegated, for example, to a standard complete and transitive criterion that picks from the shortlist. Such a procedure still has the merit of replacing the computation of explicit trade-offs with basic comparisons until a stage where the choice set has been suitably reduced. In the light of Remark 1, on the domain we study the behavioral restrictions imposed by the modified procedure still include Reducibility (because of the transitivity of the final criterion). ${ }^{23}$

\section{Concluding Remarks}

We have focussed especially on the most minimalist version of the model we are proposing, which attributes to the agent very weak powers of discrimination (basic lexicographic semiorders). On finite domains, this version is coextensive with a natural restriction of the seemingly far more general sequentially rationalizable choice model of Manzini and Mariotti (2007). On broader domains, the model restricts choice data more narrowly than even a stripped down version of sequential rationalizability (rational shortlist methods).

The Reducibility condition delimits exactly the restrictions on choice behavior that our theory implies. The weakening of Reducibility we study illustrates the additional behaviors admitted by sequential rationalizability tout court.

\footnotetext{
${ }^{22}$ We are grateful to Gil Riella for providing us with an example. As the example is rather lengthy, we omit it from the paper; however, details are available from the authors upon request.

${ }^{23}$ For a concrete illustration, consider the case of choice under uncertainty. A class of decision criteria that has gained attention because of their applicability to realistic situations is that of "quantile maximization" (see Rostek 2010). Such criteria generalize classical criteria such as maximin by allowing the use of any other quantile of the induced distribution, instead of the worst outcome. For the reasons we discussed, however, it is hard to imagine that a decision maker ignores any other aspect of a gamble except, say, its median utility. Rather, if the difference in the median outcomes of two gambles is not too large, he is likely to look at some other quantile, and so on. This procedure, which sequentially uses quantiles of interest to discard gambles, is likely to achieve a significant reduction of a choice set. After that reduction, the use of a cardinal, mean-based criterion to single out a gamble may be far more appealing, because its lack of robustness (which justifies the use of ordinal criteria first) becomes less of a drawback in small sets.
} 
While we argue that Reducibility has more than a whiff of plausibility, we have eschewed defending it as an a priori compelling property of bounded rationality. The appeal of the theory stems mostly from its psychological basis, its tractability, and its testability. Our main aim was to extend Tversky's idea into a model of choice and to tease out the observable implications of the model, in the spirit of the revealed preference approach (see Caplin 2008, Gul and Pesendorfer 2008, Rubinstein and Salant 2008 for methodological discussions of this issue). Reducibility is an easily interpretable and operationally workable concept (as demonstrated by our workouts) and, as such, we believe it fulfills this role. Our approach is thus in the same spirit as a recent body of work that seeks to characterize models of boundedly rational choice in terms of direct axioms on choice behavior (e.g., Masatlioglu and Ok 2005, 2006, Cherepanov et al. 2008, Masatlioglu and Nakajima 2011, Tyson 2008, Masatlioglu et al. forthcoming, Salant and Rubinstein 2008, Eliaz et al. 2009, in addition to those already discussed).

The present work is also related to the "checklist" model of choice in Mandler et al. (2012). In that model, an agent goes through an ordered checklist of properties (unary relations), at each step eliminating the alternatives that do not have the specified property. For example, the agent who wishes to buy a house looks first for houses in a certain location, then for those in that location with a minimum square footage, and so on until a final selection is made. A choice by basic lexicographic semiorder could be interpreted as a weakening of a choice by checklist, in which the membership of a property is allowed to have three values instead of only two. On this interpretation, $f_{i}(x)=1$ (resp., $f_{i}(x)=-1$ ) means that $x$ definitely has (resp., does not have) property $i$, while $f_{i}(x)=0$ means that $x$ neither fully has nor fully does not have property $i$ (it falls in a "grey area" or "is neutral" with respect to that property). For example, a house's location may neither be entirely convenient (e.g., close to both spouses' workplaces) nor entirely inconvenient (far from both spouses' workplaces).

Because (on certain domains) choosing by checklist is exactly equivalent to maximizing a utility function (as shown in Mandler et al. 2012), a choice by lexicographic semiorder can also be seen as a versatile but minimal departure from the standard model of rational choice.

\section{Appendix}

It is instructive to see how the algorithm to construct the rationales of Theorem 2 works. We use an example provided by Apesteguia and Ballester (2010). The grand set of alternatives is $X=\{\alpha, \beta, \gamma, \delta, \varepsilon, \varphi\}$. The inverse image of the choice function (i.e., the collection of sets from which each alternative is chosen) is

$$
c^{-1}(\alpha)=\left\{\begin{array}{l}
\{\alpha, \beta, \gamma, \delta, \varepsilon\} \\
\{\alpha, \beta, \gamma, \varepsilon\},\{\alpha, \beta, \gamma, \delta\},\{\alpha, \beta, \delta, \varepsilon\},\{\alpha, \gamma, \delta, \varepsilon\}, \\
\{\alpha, \beta, \delta\},\{\alpha, \delta, \varepsilon\},\{\alpha, \beta, \gamma\},\{\alpha, \beta, \varepsilon\},\{\alpha, \gamma, \varepsilon\}, \\
\{\alpha, \beta\},\{\alpha, \varepsilon\},\{\alpha, \delta\}
\end{array}\right\}
$$




$$
\begin{aligned}
& c^{-1}(\beta)=\left\{\begin{array}{l}
\{\beta, \gamma, \delta, \varepsilon, \varphi\}, \\
\{\beta, \gamma, \delta, \varepsilon\},\{\beta, \delta, \varepsilon, \varphi\},\{\beta, \gamma, \varepsilon, \varphi\}, \\
\{\beta, \gamma, \delta\},\{\beta, \delta, \varepsilon\},\{\beta, \gamma, \varepsilon\},\{\beta, \varepsilon, \varphi\}, \\
\{\beta, \delta\},\{\beta, \gamma\},\{\beta, \varepsilon\}
\end{array}\right\} \\
& c^{-1}(\gamma)=\left\{\begin{array}{l}
\{\gamma, \delta, \varepsilon, \varphi\},\{\alpha, \gamma, \delta, \varphi\}, \\
\{\alpha, \gamma, \varphi\},\{\alpha, \gamma, \delta\},\{\gamma, \delta, \varepsilon\},\{\gamma, \delta, \varphi\}, \\
\{\alpha, \gamma\},\{\gamma, \delta\},\{\gamma, \varphi\}
\end{array}\right\} \\
& c^{-1}(\delta)=\{\{\beta, \delta, \varphi\},\{\delta, \varepsilon, \varphi\},\{\delta, \varepsilon\},\{\delta, \varphi\}\} \\
& c^{-1}(\varepsilon)=\left\{\begin{array}{l}
X,\{\alpha, \beta, \gamma, \varepsilon, \varphi\},\{\alpha, \beta, \delta, \varepsilon, \varphi\},\{\alpha, \delta, \gamma, \varepsilon, \varphi\}, \\
\{\alpha, \beta, \varepsilon, \varphi\},\{\alpha, \gamma, \varepsilon, \varphi\},\{\alpha, \delta, \varepsilon, \varphi\}, \\
\{\alpha, \varepsilon, \varphi\},\{\gamma, \varepsilon, \varphi\}, \\
\{\gamma, \varepsilon\},\{\varepsilon, \varphi\}
\end{array}\right\} \\
& c^{-1}(\varphi)=\left\{\begin{array}{l}
\{\alpha, \beta, \delta, \gamma, \varphi\}, \\
\{\alpha, \beta, \gamma, \varphi\},\{\beta, \gamma, \delta, \varphi\},\{\alpha, \beta, \delta, \varphi\}, \\
\{\alpha, \beta, \varphi\},\{\beta, \gamma, \varphi\},\{\alpha, \delta, \varphi\}, \\
\{\alpha, \varphi\},\{\beta, \varphi\}
\end{array}\right\} .
\end{aligned}
$$

The base relation $P_{c}=\{(a, b) \in X \times X \mid a=c(\{a, b\})\}$ is thus

$$
P_{c}=\left\{\begin{array}{l}
(\alpha, \beta),(\alpha, \varepsilon),(\alpha, \delta),(\delta, \varepsilon),(\delta, \varphi),(\beta, \delta),(\beta, \gamma),(\beta, \varepsilon) \\
(\gamma, \alpha),(\gamma, \delta),(\gamma, \varphi),(\varepsilon, \gamma),(\varepsilon, \varphi),(\varphi, \alpha),(\varphi, \beta)
\end{array}\right\}
$$

If the rationales $P_{i}$ and the collections $\mathcal{C}_{i-1}$ are built according to the algorithm in the proof of Theorem 2, obviously it can never be $(a, b) \in P_{c} \cap P_{i}$ for any $a$ and $b$ such that $b$ is chosen from some $S \in \mathcal{C}_{i-1}$ that also contains $a$. Consequently, we are going to construct the rationales by first ruling out as potential members of $P_{i}$ all such pairs; then we verify whether the residual subcollection of pairs in $P_{c}$ that have not yet been "allocated" to any previous rationale $P_{j}, j<i$, satisfy the requirement in the Weak Reducibility axiom, removing more pairs if necessary until we have the largest collection that satisfies the axiom.

Beginning with $\mathcal{C}_{0}=\Sigma$, inspection of the inverse images reveals that the only candidate pairs are $(\alpha, \beta),(\alpha, \delta),(\beta, \gamma),(\gamma, \delta),(\varepsilon, \varphi)$, and $(\varphi, \alpha)$, since for all other pairs $(a, b) \in P_{c}$ it is always the case that $b$ is chosen in some set where $a$ is present.

However, $\delta$ is also the only alternative such that, when it is removed from sets that also contain $\alpha$, leaves choice unchanged. To see this, observe that $(\alpha, \beta) \notin P_{1}$, since, e.g., $\alpha=c(\{\alpha, \beta, \gamma\}) \neq c(\{\alpha, \gamma\})=\gamma ;(\beta, \gamma) \notin P_{1}$, since, e.g., $\varphi=c(\{\beta, \gamma, \delta, \varphi\}) \neq$ $c(\{\beta, \delta, \varphi\})=\delta ;(\gamma, \delta) \notin P_{1}$, since, e.g., $\gamma=c(\{\gamma, \delta, \varepsilon\}) \neq c(\{\gamma, \varepsilon\})=\varepsilon ;(\varepsilon, \varphi) \notin P_{1}$, since, e.g., $\varepsilon=c(\{\alpha, \varepsilon, \varphi\}) \neq c(\{\alpha, \varepsilon\})=\alpha$; and $(\varphi, \alpha) \notin P_{1}$, since, e.g., $\varepsilon=c(\{\alpha, \beta, \varepsilon, \phi\}) \neq$ $c(\{\beta, \varepsilon, \phi\})=\beta$.

Consequently,

$$
P_{1}=\{(\alpha, \delta)\} .
$$


The domain thus reduces from $\mathcal{C}_{0}$ to $\mathcal{C}_{1}$ as indicated in the display that follows (simply remove all sets containing $\alpha$ and $\delta$ ), where observe that the first line is a subcollection of $c^{-1}(\alpha)$, the second line is a subcollection of $c^{-1}(\beta)$, and so on:

$$
\mathcal{C}_{1}=\left\{\begin{array}{l}
\{\alpha, \beta, \gamma, \varepsilon\},\{\alpha, \beta, \gamma\},\{\alpha, \beta, \varepsilon\},\{\alpha, \gamma, \varepsilon\},\{\boldsymbol{\alpha}, \boldsymbol{\beta}\},\{\alpha, \varepsilon\} \\
\{\beta, \gamma, \delta, \varepsilon, \varphi\},\{\beta, \gamma, \delta, \varepsilon\},\{\beta, \delta, \varepsilon, \varphi\},\{\beta, \gamma, \varepsilon, \varphi\} \\
\{\beta, \gamma, \delta\},\{\beta, \delta, \varepsilon\},\{\beta, \gamma, \varepsilon\},\{\beta, \varepsilon, \varphi\},\{\beta, \delta\},\{\boldsymbol{\beta}, \boldsymbol{\gamma}\},\{\beta, \varepsilon\} \\
\{\gamma, \delta, \varepsilon, \varphi\},\{\alpha, \gamma, \varphi\},\{\gamma, \delta, \varepsilon\},\{\gamma, \delta, \varphi\},\{\alpha, \gamma\},\{\gamma, \boldsymbol{\delta}\},\{\gamma, \varphi\} \\
\{\beta, \delta, \varphi\},\{\delta, \varepsilon, \varphi\},\{\boldsymbol{\delta}, \boldsymbol{\varepsilon}\},\{\delta, \varphi\} \\
\{\alpha, \beta, \gamma, \varepsilon, \varphi\},\{\alpha, \beta, \varepsilon, \varphi\},\{\alpha, \gamma, \varepsilon, \varphi\},\{\alpha, \varepsilon, \varphi\},\{\gamma, \varepsilon, \varphi\},\{\gamma, \varepsilon\},\{\boldsymbol{\varepsilon}, \boldsymbol{\varphi}\} \\
\{\alpha, \beta, \gamma, \varphi\},\{\beta, \gamma, \delta, \varphi\},\{\alpha, \beta, \varphi\},\{\beta, \gamma, \varphi\},\{\boldsymbol{\alpha}, \boldsymbol{\varphi}\},\{\beta, \varphi\}
\end{array}\right\} .
$$

Next, observe that $\alpha$ and $\varphi$ are chosen in the presence of $\gamma$, so that our algorithm prescribes $(\gamma, \alpha) \notin P_{2}$ and $(\gamma, \varphi) \notin P_{2}$. Moreover, $\beta$ is chosen in the presence of $\varphi ; \gamma$ is chosen in the presence of $\varepsilon ; \delta$ and $\varepsilon$ are chosen in the presence of $\beta$; $\varepsilon$ is chosen in the presence of $\alpha$; and $\varphi$ is chosen in the presence of $\delta$. This leaves only $(\alpha, \beta),(\beta, \gamma)$, $(\gamma, \delta),(\delta, \varepsilon),(\varepsilon, \varphi)$, and $(\varphi, \alpha)$ as potential members of $P_{2}$ (which appear in boldface in the above display), and it is easy to verify that indeed the whole collection of "candidate pairs"

$$
P_{2}=\{(\alpha, \beta),(\beta, \gamma),(\gamma, \delta),(\delta, \varepsilon),(\varepsilon, \varphi),(\varphi, \alpha)\}
$$

is such that $c(S)=c\left(S \backslash \bigcup_{i: x_{i} \in S} y_{i}\right)$ for all $S \in \mathcal{C}_{1}$ as in the definition of Weak Reducibility. Note also that Reducibility fails on the collection $\mathcal{C}_{1}$ : no set contains $\alpha$ and $\delta$, and by the same considerations contained in the previous paragraphs, the only pairs of alternatives that might satisfy Reducibility are $\{\alpha, \beta\},\{\beta, \gamma\},\{\gamma, \delta\},\{\delta, \varepsilon\},\{\varepsilon, \varphi\}$, and $\{\varphi, \alpha\}$. However, none of them does: first of all, because all these binary sets are in $\mathcal{C}_{1}$, the "losing" alternative must be the one that is not chosen in pairwise sets; in addition, $\left(x_{2}, y_{2}\right) \notin\{(\alpha, \beta),(\beta, \gamma),(\gamma, \delta),(\varepsilon, \varphi),(\varphi, \alpha)\}$, since the same sets for which $c(S) \neq c\left(S \backslash\left\{y_{2}\right\}\right)$ when checking $\mathcal{C}_{0}$ are also in $\mathcal{C}_{1}$, while $x_{2}, y_{2} \neq \delta$, $\varepsilon$, since, e.g., $\beta=c(\{\beta, \gamma, \delta, \varepsilon, \varphi\}) \neq c(\{\beta, \gamma, \delta, \varphi\})=\varphi$.

Going back to our algorithm, the construction of $P_{2}$ yields

$$
\mathcal{C}_{2}=\left\{\begin{array}{c}
\{\alpha, \gamma, \varepsilon\},\{\boldsymbol{\alpha}, \boldsymbol{\varepsilon}\} \\
\{\beta, \delta\},\{\boldsymbol{\beta}, \boldsymbol{\varepsilon}\} \\
\{\alpha, \gamma\},\{\boldsymbol{\gamma}, \boldsymbol{\varphi}\} \\
\{\beta, \delta, \varphi\},\{\boldsymbol{\delta}, \boldsymbol{\varphi}\} \\
\{\boldsymbol{\gamma}, \boldsymbol{\varepsilon}\} \\
\{\boldsymbol{\beta}, \boldsymbol{\varphi}\}
\end{array}\right\} .
$$

For the next step, we note that $\delta$ is chosen in the presence of $\beta$; $\alpha$ is chosen in the presence of $\gamma$. So one can verify that all together the remaining candidate pairs provide a suitable $P_{3}$, that is,

$$
P_{3}=\{(\alpha, \varepsilon),(\varepsilon, \gamma),(\beta, \varepsilon),(\delta, \varphi),(\varphi, \beta),(\gamma, \varphi)\}
$$


As a consequence, the subdomain reduces to

$$
\mathcal{C}_{3}=\{\{\beta, \delta\},\{\alpha, \gamma\}\}
$$

so that we can build the final rationale

$$
P_{4}=\{(\beta, \delta),(\gamma, \alpha)\} .
$$

It is straightforward to double check that $P_{1}, P_{2}, P_{3}$, and $P_{4}$ so defined sequentially rationalizes $c$.

\section{REFERENCES}

Apesteguia, José and Miguel A. Ballester (2010), “Choice by sequential procedures.” Unpublished paper, Universitat Autonoma de Barcelona and Universitat Pompeu Fabra. [1, $3,8,10,16,18]$

Binmore, K., A. Voorhoeve, and B. Wallace (2008), "Similarity-based decision-making in moral decisions: Experimental results.” Mimeo, London School of Economics. [4]

Caplin, Andrew (2008), "Economic theory and psychological data: Bridging the divide." In The Foundations of Positive and Normative Economics (Andrew Caplin and Andrew Schotter, eds.), 336-372, Oxford University Press, Oxford. [18]

Cherepanov, Vadim, Timothy Feddersen, and Alvaro Sandroni (2008), "Rationalization." Unpublished paper, Northwestern University. [18]

Ehlers, Lars and Yves Sprumont (2008), "Weakened warp and top-cycle choice rules." Journal of Mathematical Economics, 44, 87-94. [5]

Eliaz, Kfir, Michael Richter, and Ariel Rubinstein (2009), "An étude in choice theory: Choosing the two finalists." Unpublished paper, Brown University, New York University, and Tel Aviv University. [18]

Fishburn, Peter C. (1970), "Intransitive indifference with unequal indifference intervals." Journal of Mathematical Psychology, 7, 144-149. [4]

Fishburn, Peter C. (1985), Interval Orders and Interval Graphs: A Study of Partially Ordered Sets. Wiley, New York. [4]

Gigerenzer, Gerd and Peter M. Todd (1999), "Fast and frugal heuristics: The adaptive toolbox." In Simple Heuristics That Make Us Smart (Gerd Gigerenzer and Peter M. Todd, eds.), 3-34, Oxford University Press, New York. [1]

Gul, Faruk and Wolfgang Pesendorfer (2008), “The case for mindless economics.” In The Foundations of Positive and Normative Economics (Andrew Caplin and Andrew Schotter, eds.), 3-42, Oxford University Press, Oxford. [18]

Houy, Nicolas and Koichi Tadenuma (2009), "Lexicographic compositions of multiple criteria for decision making." Journal of Economic Theory, 144, 1770-1782. [1] 
Kalandrakis, Tasos (2010), "Rationalizable voting.” Theoretical Economics, 5, 93-125. [3, 11]

Kohli, Rajeev and Kamel Jedidi (2007), "Representation and inference of lexicographic preference models and their variants.” Marketing Science, 26, 380-399. [1]

Leland, Jonathan W. (1994), "Generalized similarity judgments: An alternative explanation for choice anomalies." Journal of Risk and Uncertainty, 9, 151-172. [1, 2]

Lombardi, Michele (2008), “Uncovered set choice rules.” Social Choice and Welfare, 31, 271-279. [5]

Luce, R. Duncan (1956), "Semiorders and a theory of utility discrimination." Econometrica, 24, 178-191. [4]

Luce, R. Duncan and Howard Raiffa (1957), Games and Decisions. Wiley, New York. [12]

Mandler, Michael (2009), "Rational agents are the quickest.” Unpublished paper, Royal Holloway University. [10]

Mandler, Michael, Paola Manzini, and Marco Mariotti (2012), "A million answers to twenty questions: Choosing by checklist." Journal of Economic Theory, 147, 71-92. [3, 18]

Manzini, Paola and Marco Mariotti (2006), "A vague theory of choice over time." Advances in Theoretical Economics, 6, Art. 6. [2]

Manzini, Paola and Marco Mariotti (2007), "Sequentially rationalizable choice.” American Economic Review, 97, 1824-1839. [1, 3, 6, 8, 16, 17]

Masatlioglu, Yusufcan, Daisuke Nakajima, and Erkut Y. Ozbay (forthcoming), "Revealed attention." American Economic Review. [18]

Masatlioglu, Yusufcan and Daisuke Nakajima (2011), “Choice by iterative search.” Unpublished paper, University of Michigan. [18]

Masatlioglu, Yusufcan and Efe Ok (2005), “Rational choice with status quo bias.” Journal of Economic Theory, 121, 1-29. [18]

Masatlioglu, Yusufcan and Efe Ok (2006), "Reference-dependent procedural decision making.” Unpublished paper, New York University. [18]

Medina, Barak, Shlomo Naeh, and Uzi Segal (2011), “Ranking ranking rules.” Unpublished paper, Boston College. [1]

Rostek, Marzena (2010), “Quantile maximization in decision theory.” Review of Economic Studies, 77, 339-371. [17]

Rubinstein, Ariel (1988), "Similarity and decision making under risk (is there a utility theory resolution to the Allais paradox?)." Journal of Economic Theory, 46, 145-153. [1, 2, 4]

Rubinstein, Ariel and Yuval Salant (2008), "Some thoughts on the principle of revealed preference." In The Foundations of Positive and Normative Economics (Andrew Caplin and Andrew Schotter, eds.), 116-124, Oxford University Press, Oxford. [18] 
Salant, Yuval and Ariel Rubinstein (2008), “( $A, f)$ : Choice with frames.” Review of Economic Studies, 75, 1287-1296. [18]

Tadenuma, Koichi (2002), "Efficiency first or equity first? Two principles and rationality of social choice." Journal of Economic Theory, 104, 462-472. [1]

Tadenuma, Koichi (2005), "Egalitarian-equivalence and the Pareto principle for social preferences." Social Choice and Welfare, 24, 455-473. [1]

Tversky, Amos (1969), "Intransitivity of preferences." Psychological Review, 76, 31-48. [1, $2,4,6]$

Tversky, Amos (1972), "Elimination by aspects: A theory of choice." Psychological Review, 79, 281-299. [1]

Tyson, Christopher J. (2008), "Cognitive constraints, contraction consistency, and the satisficing criterion." Journal of Economic Theory, 138, 51-70. [18]

Yee, Michael, Ely Dahan, John R. Hauser, and James Orlin (2007), “Greedoid-based noncompensatory inference." Marketing Science, 26, 532-549. [1]

Submitted 2009-11-16. Final version accepted 2010-11-4. Available online 2010-11-4. 